\title{
Les socialistes et la Première Guerre Mondiale: quelques éléments du débat entre socialistes au moment du passage de l'opposition à la guerreà I"union sacrée"
}

Os socialistas e a Primeira Guerra Mundial: alguns elementos do debate entre os socialistas, no momento da passagem da oposição à guerra para a "união sagrada"

The Socialists and the First World War : some elements of the debate between socialists at the time of passage of the opposition to the war to the "sacred union"

Jorge MagasichAirola*

\section{Resumo}

Pendant son quart de siècle d'existence, l'Internationale ouvrière socialiste (IOS ou Deuxième Internationale), a exprimé systématiquement des positions opposées au militarisme et à la guerre, sauf lors des tout derniers jours précédant le conflit. D'une manière peut-être surprenante, les grands partis socialistes abandonnèrent leurs positions historiques et se rallièrent à $\mathrm{l}^{\prime}$ "union sacrée", soutenant chacun l'effort de guerre de leur pays. Sauf quelques exceptions. Dans cet article nous souhaitons retracer tout d'abord certains aspects du positionnement historique des socialistes à propos de la guerre; nous ferons ensuite le récit de leur revirement, pour terminer avançant quelques explications.

Mot clés: Social-démocratie. Union-sacrée. Antimilitarisme. Deuxième internationale.

Doctor en historia, arte y arqueología por la Universidad Libre de Bruselas(ULB), 2007. Desde 1989, conferenciante en los ciclos de formación general organizados por la Coopération technique belge (CTB); conferencia: Historia y sociología de América latina. Desde 1980, encargado del programa radiofónico Historia de América en la radio Campus de la ULB. Colaborador de la revistas: Revue Nouvelle, Cahiers du Centre d'études Latino--américains de l'Institut de Sociologie (ULB), Panorámica, El Clarín digital y otras. E-mail: jorge.magasich@skynet.be

Recebido em 30/07/2014 - Aprovado em 10/10/2014 http://dx.doi.org/10.5335/hdtv.14n.2.4576 
Pratiquement toutes les prises de positions des partis sociaux-démocrates organisés dans l'IOS, jusqu'en juillet 1914, expriment invariablement les principes antimilitaristes et anti-bellicistes et proclament haut et fort être résolus à organiser une grève ouvrière internationale pour éviter la "barbarie de la guerre". Ils en ont sans doute les moyens. Les socialistes, en effet, disposent d'une influence significative dans plusieurs pays européens (Angleterre, Autriche, Belgique, Pays-Bas, Russie, Serbie...), ils sont un grand parti en France et le premier parti d'Allemagne.

En France, plusieurs courants socialistes s'étaient unifiés en 1902 dans la Section française de l'internationale ouvrière (SFIO) autour des personnalités comme Aristide Briand, René Viviani et Jean Jaurès. En 1904, ils publient le quotidien L'Humanité, dirigé par ce dernier jusqu'à sa mort.

L'un de ces grands combats de la SFIO sera l'opposition au projet de loi qui veut faire passer de deux à trois ans la durée du service militaire obligatoire. Malgré les articles de Jean Jaurès et ses éloquents discours lors d'un important meeting en plein air au Pré-Saint-Gervais, suivi quelques jours plus tard d'une colossale manifestation de 150.000 personnes le 13 juillet 1913, la loi est adoptée. Lors de controverses, la figure de Jean Jaurès devient le symbole l'opposition à la guerre, mais aussi la cible haïe du bloc nationaliste "patriote", qui tire à boulets-rouges contre celui qui est accusé de "trahir la patrie" et d'être "l'ami du Kaiser".

Malgré cela, la SFIO fait une percée aux élections parlementaires d'avril 1914 (à trois mois du déclanchement de la guerre), devenant le deuxième parti après les radicaux, avec 1,4 million de votes (17\%).
En 1912, rappelle Broué, le Deuxième Reich allemand est toujours un empire où les junkers, les aristocrates fonciers, conservent une puissance considérable. L'armée et la bureaucratie continuent à bénéficier de privilèges, entre autres celui du suffrage censitaire.

Si, depuis 1869, le Reichstag est élu au suffrage universel masculin (à partir de 25 ans), la plupart des députés des Landtag (parlements des États), notamment celui de Prusse, sont élus selon un système censitaire de "trois classes". En 1849, la première classe (tiers supérieur de l'imposition) ne comprenait que $4,7 \%$ de la population; la deuxième $12,7 \%$, et la troisième $82,6 \%$. Chaque classe élisait cependant le même nombre de représentants, système qui surreprésente les plus fortunés. A Essen par exemple, le riche industriel Alfred Krupp était le seul électeur de la première classe.

La croissance économique et industrielle fulgurante du puissant empire lui permet de dépasser la production britannique en divers domaines dès 1890, devenant la deuxième puissance industrielle mondiale après les Etats-Unis. Une telle position stimule les ambitions expansionnistes d'une partie de cette nouvelle bourgeoisie. Mais, à cette date, le partage du monde entre les empires, particulièrement celui de l'Afrique, était terminé. Le Reich réussi à accaparer la Namibie, le Cameroun, le Togo, la Tanzanie et le Rwanda-Burundi. Toutefois, cet empire colonial reste bien plus "petit" que l'anglais ou le français. Arrivé plus tard au partage colonial, l'impérialisme allemand revendique un repartage du "gâteau". Ce qui accroit le risque de guerre. 
Les nouvelles industries sont à l'origine d'un prolétariat nombreux et sans doute le mieux organisé au monde. Il est, selon Broué, "relativement instruit, familiarisé avec la technique et les machines, ayant le sens $d u$ travail collectif et des responsabilités, le goût de l'organisation". Il s'agit d'une classe sociale consciente que la "solidarité fait d'eux une force capable de transformer leur vie", et même celle des « petits-bourgeois » qu'ils considèrent comme leurs alliés. Le prolétariat allemand est le premier à bénéficier d'une assurance contre les accidents du travail.

L'organisation des travailleurs allemands est, incontestablement, le Sozialdemokratische Partei Deutschlands (SPD). Sa progression électorale lors des 13 élections au Reichstag organisées entre 1871 et 1912, est éloquente. En quatre décennies il passe d'un modeste 3,2\% à un imposant 34,8\%. En 1912, le SPD est, en même temps, le principal parti de l'Allemagne et le principal parti socialiste de l'époque.

Avec plus d'un millions d'adhérents en 1914 et quelque milliers de permanents, le SPD encadre deux millions de travailleurs syndiqués et un réseau de mouvements de femmes et de jeunes, de bibliothèques, d'universités populaires et de maisons d'éditions qui publient livres, revues, et 90 quotidiens. Il a 110 députés au Reichstag, 220 dans les Landtag et 2.886 élus municipaux. Ce puissant parti était, en apparence, profondément anti-belliciste, à l'instar des autres partis socialistes. ${ }^{1}$ La tradition pacifiste était forte. Déjà en 1870, les dirigeants du SPD, Wilhelm Liebknecht et August Bebel, avaient refusé de voter les crédits de guer- re qualifiant la guerre franco-prussienne de "guerre dynastique". 2

Cependant, le pouvoir de l'appareil syndical et celui des permanents politiques s'accroit notablement, en même temps que la tendance à s'installer dans des routines conformistes, voire dans un esprit conservateur. 
Résultats des élections au Reichstag, de 1871 à 1912 (en \% des voix exprimées)

\begin{tabular}{|c|c|c|c|c|c|c|c|c|}
\hline Année & $\begin{array}{c}\text { Nationaux- } \\
\text { libéraux }\end{array}$ & $\begin{array}{c}\text { Libérauxde } \\
\text { gauche }\end{array}$ & $\begin{array}{c}\text { Conserva- } \\
\text { teurs }\end{array}$ & Centre & $\begin{array}{c}\text { Sociaux- } \\
\text { démocrates }\end{array}$ & Antisémites & Divers & $\begin{array}{c}\text { Participation } \\
\text { en \% }\end{array}$ \\
\hline 1871 & 30,1 & 9,3 & 23,0 & 18,6 & 3,2 & - & 15,8 & 50,7 \\
1874 & 29,7 & 9,0 & 14,1 & 27,9 & 6,8 & - & 12,4 & 60,8 \\
1877 & 27,2 & 8,5 & 17,6 & 24,8 & 9,1 & - & 10,1 & 60,3 \\
1878 & 23,1 & 7,8 & 26,6 & 23,1 & 7,6 & - & 9,0 & 63,1 \\
1881 & 14,7 & 23,1 & 23,7 & 23,2 & 6,1 & - & 9,1 & 56,1 \\
1884 & 17,6 & 19,3 & 22,1 & 22,6 & 9,7 & - & 8,7 & 60,3 \\
1887 & 22,3 & 14,1 & 25,0 & 20,1 & 10,1 & 0,2 & 8,2 & 77,2 \\
1890 & 16,3 & 18,0 & 19,1 & 18,6 & 19,8 & 0,7 & 8,6 & 71,2 \\
1893 & 13,0 & 14,8 & 19,3 & 19,1 & 23,4 & 3,5 & 7,7 & 72,2 \\
1898 & 12,5 & 11,1 & 15,5 & 18,8 & 27,2 & 3,3 & 10,6 & 67,7 \\
1903 & 13,9 & 9,3 & 13,5 & 19,8 & 31,7 & 2,6 & 9,5 & 75,3 \\
1907 & 14,5 & 10,9 & 13,6 & 19,4 & 28,9 & 3,9 & 8,8 & 84,3 \\
1912 & 13,6 & 12,3 & 12,2 & 16,4 & 34,8 & 2,9 & 7,7 & 84,5 \\
\hline
\end{tabular}

Fonte: http://www.bundestag.de/blob/189798/6f1db854d5631be208d26982bbd36f7d/elections_imperiale-data.pdf.

\section{D- L'Internationale ouvrière socialiste contre la guerre. En apparence}

Les années qui précédent la "Grande guerre" sont marquées par deux fait majeurs. D'une part, le continent européen vivait quatre décennies de paix après la guerre franco-prussienne de 1870, mais, de l'autre, les guerres étaient une constante dans le reste du monde pendant la même période.

Elles sont nombreuses. Le Japon, devenu une grande puissance en quête de colonies sur le continent asiatique, fait la guerre à la Chine (1894-95) et plus tard à la Russie (1904-07); les Etats-Unis déclarent la guerre à l'Espagne et s'emparent de ses trois der- nières colonies en 1898 (Cuba, Porto-Rico et Philippines); l'Angleterre fait la guerre aux Boers du Transvaal, en Afrique australe (1899-1902); plusieurs puissances européennes se sont emparées de territoires chinois; l'Allemagne fait la guerre aux Hereros de l'actuelle Namibie, perpétrant le premier génocide du XX $X^{\mathrm{e}}$ s.; la Russie intervient contre la Perse (actuel Iran) et la France au Maroc.

Le risque que tels conflits gagnent l'Europe était présent dans les analyses de l'IOS. En 1907, à Stuttgart, elle dénonce le danger de guerre et appelle à "utiliser, avec toutes leurs forces, la crise économique pour soulever les masses et précipiter la chute de la domination de la classe capitaliste". 


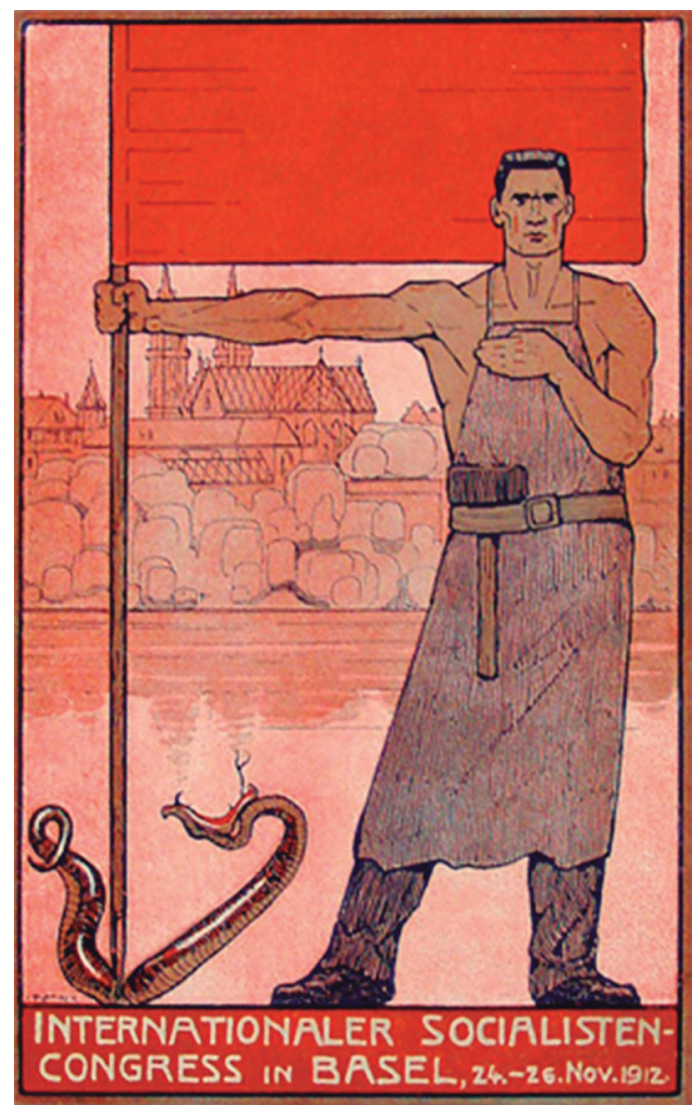

Affiche annonçant le congrès de l'IOS à Bâle (nov. 1912): la classe ouvrière écrase la guerre.

Conclusions du congrès: "Attitude des partis socialistes en cas de guerre": "En cas de déclaration de guerre, les classes laborieuses des pays intéressés ainsi que leurs représentants parlementaires seront tenus de mobiliser toutes leurs forces pour empêcher le déclenchement des hostilités [...] Au cas où la guerre éclaterait en dépit de nos efforts, elles seront tenues de militer pour une fin rapide des hostilités et de tenter de toutes leurs forces d'exploiter la crise économique et politique provoquée par la guerre afin de soulever le peuple et d'accélérer ainsi l'abolition de la domination de la classe capitaliste"3.

Cette menace d'un conflit majeur à l'échelle continentale se rapproche en 1911, quand l'Italie entre en guerre contre la Turquie et réussit à s'emparer de l'actuelle Libye et du Dodécanèse. Elle se précise en 1912 quand le Monténégro, allié à la Bulgarie, à la Grèce et à la Serbie, déclare la guerre à la
Turquie, ce qui déclenche la première Guerre balkanique. Quelque mois plus tard, les vainqueurs de la Turquie se disputent entre eux et partent tous en guerre contre la Bulgarie: c'est la deuxième Guerre balkanique. Cette fois on se bat en Europe.

Alertée par la proximité du conflit, l'Internationale ouvrière socialiste réunit à Bâle 555 délégués venus de 23 pays pour analyser le danger et étudier les manières de le contrer. Ils votent à l'unanimité un manifeste rédigé par le Bureau qui proclame que la guerre, prochaine, ne pourrait avoir qu'un caractère impérialiste et confirme la position adoptée au congrès antérieur : organiser la lutte des ouvriers contre la guerre. Si la guerre éclate, prévient l'IOS, elle sera suivie d'une révolte sociale (voir encadré).

Le Français Jean Jaurès, intellectuel de grande envergure, orateur exceptionnel, directeur du quotidien L'Humanité et l'un des principaux dirigeants de l'IOS, perçoit que le danger de guerre se précise. Il propose une "organisation vraiment populaire de la défense nationale" basée sur la "nation armée":

arracher la patrie aux maquignons de la patrie, aux castes de militarisme et aux bandes de la finance ; permettre à toutes les nations le développement indéfini dans la démocratie et la paix. ${ }^{4}$

Une telle prise de position lui vaudra les foudres des "patriotes", qui l'accusent de trahison. Les invectives contre Jaurès se font virulentes.

Le 25 juillet 1914, la veille de l'ultimatum de l'Autriche-Hongrie à la Serbie, Jaurès prononce son avant-dernier discours à Vaise, dans les faubourgs de Lyon, 
pour soutenir le candidat socialiste Marius Moutet à une élection législative partielle ${ }^{5}$.

Il explique que la situation est menaçante et tragique. L'ultimatum risque d'activer, d'abord, le traité entre la Serbie et la Russie et ensuite le traité entre la Russie et la France (partiellement secret) et, d'autre part, l'alliance entre l'Autriche et l'Allemagne. Autrement dit, faire éclater la guerre à l'échelle continentale. Pour contrer cette menace contre la paix -clame-t-il- "il faudra que les prolétaires de l'Europe tentent les efforts de solidarité suprême".

Le directeur de L'Humanité analyse d'abord les responsabilités des Français "quand l'Autriche annexait la Bosnie-Herzégovine, nous n'avions pas le droit ni le moyen de lui opposer la moindre remontrance, parce que nous étions engagés au Maroc". De même avec l'Italie, quand la France lui suggérait: " $t u$ peux aller en Tripolitaine [actuelle Libye] puisque je suis au Maroc; tu peux voler à l'autre bout de la rue, puisque moi j'ai volé à l'extrémité'."

Il signale ensuite les responsabilités de la Russie, qui a créé la Bulgarie en 1877 pour avoir un accès à la mer Noire et, en contrepartie, a laissé faire l'Autriche en Bosnie, où cette dernière a imposé le catholicisme par la force.

Enfin, Jaurès rappelle comment les guerres balkaniques augurent des tragédies à venir : elles ont laissé,

dans la terre des champs de bataille, dans les fossés des chemins ou dans les lits d'hôpitaux infectés par le typhus cent mille hommes sur trois cent mille. Songez à ce que serait le désastre pour $l^{\prime}$ Europe: quatre, cinq et six armées de deux millions d'hommes. Quel massacre, quelles ruines, quelle barbarie!
Dans le Vorwaerts, ${ }^{6}$-rappelle Jaurèsnos camarades socialistes d'Allemagne s'élèvent avec indignation contre la note de l'Autriche et je crois que notre Bureau socialiste international est convoqué. Il conclut : il n'y a

[...] qu'une chance pour le maintien de la paix et le salut de la civilisation, c'est que le prolétariat rassemble toutes ses forces qui comptent un grand nombre de frères, Français, Anglais, Allemands, Italiens, Russes et que nous demandions à ces milliers d'hommes de s'unir pour que le battement unanime de leurs cœurs écarte l'horrible cauchemar.

Dans ce discours, Jaurès identifie des responsabilités partagées et équivalentes entre la "politique coloniale de la France, la politique sournoise de la Russie et la volonté brutale de l'Autriche [qui] ont contribué à créer l'état de choses horrible où nous sommes."

L'IOS réagit à l'ultimatum de l'Autriche-Hongrie à la Serbie en convoquant d'urgence, les 29 et 30 juillet, sont Bureau qui siège à Bruxelles. Cette réunion sera la dernière tentative organisée d'éviter l'embrasement général, à laquelle participent Allemands, Français, Belges, Italiens, et d'autres délégués des pays impliqués.

Lorsque le 29 juillet le Bureau se réunit à la Maison du peuple, parvient l'information que, depuis la veille, l'état de guerre est en vigueur entre l'Autriche-Hongrie et la Serbie. Pour l'instant toutefois, le conflit n'a pas dépassé le stade des déclarations; il n'y a pas encore de combats. ${ }^{7}$

Le Bureau comprend que la seule réponse encore efficace est une action internationale des travailleurs des pays impliqués. Il décide d'organiser d'urgence de grandes 
manifestations contre la guerre en Allemagne, Autriche, Belgique, France et Italie. Le point d'orgue de l'action sera une immense manifestation à Paris, le 9 août.

Le soir, les dirigeants se déplacent au Cirque royal où se tient un meeting contre la guerre organisé par le Parti ouvrier belge.
Devant une salle comble, Emile Vandervelde ouvre la séance en tant que dirigeant du parti invitant. Rosa Luxemburg est dans la salle, au nom des socialistes allemands. Jean Jaurès prononce son dernier discours. Celui-ci n'a pas été sténographié, mais l'historien Jean Stengers a pu le reconstituer à partir de plusieurs comptes-rendus.

Extraits du discours de Jean Jaurès à Bruxelles, le soir du 29 juillet 1914

"Nous ne sommes pas ici, cependant, pour nous abandonner à ces émotions mais pour mettre en commun, contre le monstrueux péril de la guerre, toutes nos forces de volonté et de raison [...] On négocie; il paraît qu'on se contentera de prendre à la Serbie un peu de son sang, et non un peu de chair (Rires); nous avons donc un peu de répit pour assurer la paix. [...]

L'Autriche-Hongrie, [...] par la brutalité de sa note, avec son mélange de violence et de jésuitisme, la coterie militaire et cléricale de Vienne semble avoir voulu passer au premier plan.

Et l'Allemagne du Kaiser, comment pourra-t-elle justifier son attitude de ces derniers jours? Si elle a connu la note austro-hongroise, elle est inexcusable d'avoir pardonné pareille démarche. Et si l'Allemagne officielle n'a pas connu la note autrichienne, que devient la prétendue sagesse gouvernementale (Rires)

Nous, socialistes français, notre devoir est simple. Nous n'avons pas à imposer à notre gouvernement une politique de paix. II la pratique. [...] Le gouvernement français est le meilleur allié de la paix de cet admirable gouvernement anglais qui a pris l'initiative de la médiation. Et il donne à la Russie des conseils de prudence et de patience. Quant à nous, c'est de notre devoir d'insister pour qu'il parle avec force à la Russie de façon qu'elle s'abstienne. Mais, si par malheur, la Russie n'en tenait pas compte, notre devoir est de dire: "Nous ne connaissons qu'un traité: celui qui nous lie à la race humaine! Nous ne connaissons pas les traités secrets!" (Ovation) Voilà notre devoir et, en l'exprimant, nous nous sommes trouvés d'accord avec les camarades d'Allemagne qui demandent à leur gouvernement de faire que l'Autriche modère ses actes. [...]

Voulez-vous que je vous dise la différence entre la classe ouvrière et la classe bourgeoise? C'est que la classe ouvrière hait la guerre collectivement, mais ne la craint pas individuellement, tandis que les capitalistes, collectivement, célèbrent la guerre, mais la craignent individuellement. (Acclamations) [...] Mais, pour les maîtres absolus, le terrain est miné. Si dans l'entraînement mécanique et dans l'ivresse des premiers combats, ils réussissent à entraîner les masses, à mesure que les horreurs de la guerre se développeraient, à mesure que le typhus achèverait l'œuvre des obus, à mesure que la mort et la misère frapperaient, les hommes dégrisés se tourneraient vers les dirigeants allemands, français, russes, italiens, et leur demanderaient: quelle raison nous donnez-vous de tous ces cadavres? Et alors, la Révolution déchaînée leur dirait: "Va-t'en, et demande pardon à Dieu et aux hommes!" (Acclamations) Le prolétariat prend conscience de sa sublime mission. Et le 9 août, des millions et des millions de prolétaires, par l'organe de leurs délégués, viendront affirmer à Paris l'universelle volonté de paix de tous les peuples. Longues ovations. Toute la salle, debout, acclame Jaurès.

La seule photo conservée du meeting au Cirque royal. Jaurès est au milieu (c'est sans doute sa dernière photo $)^{8}$ 


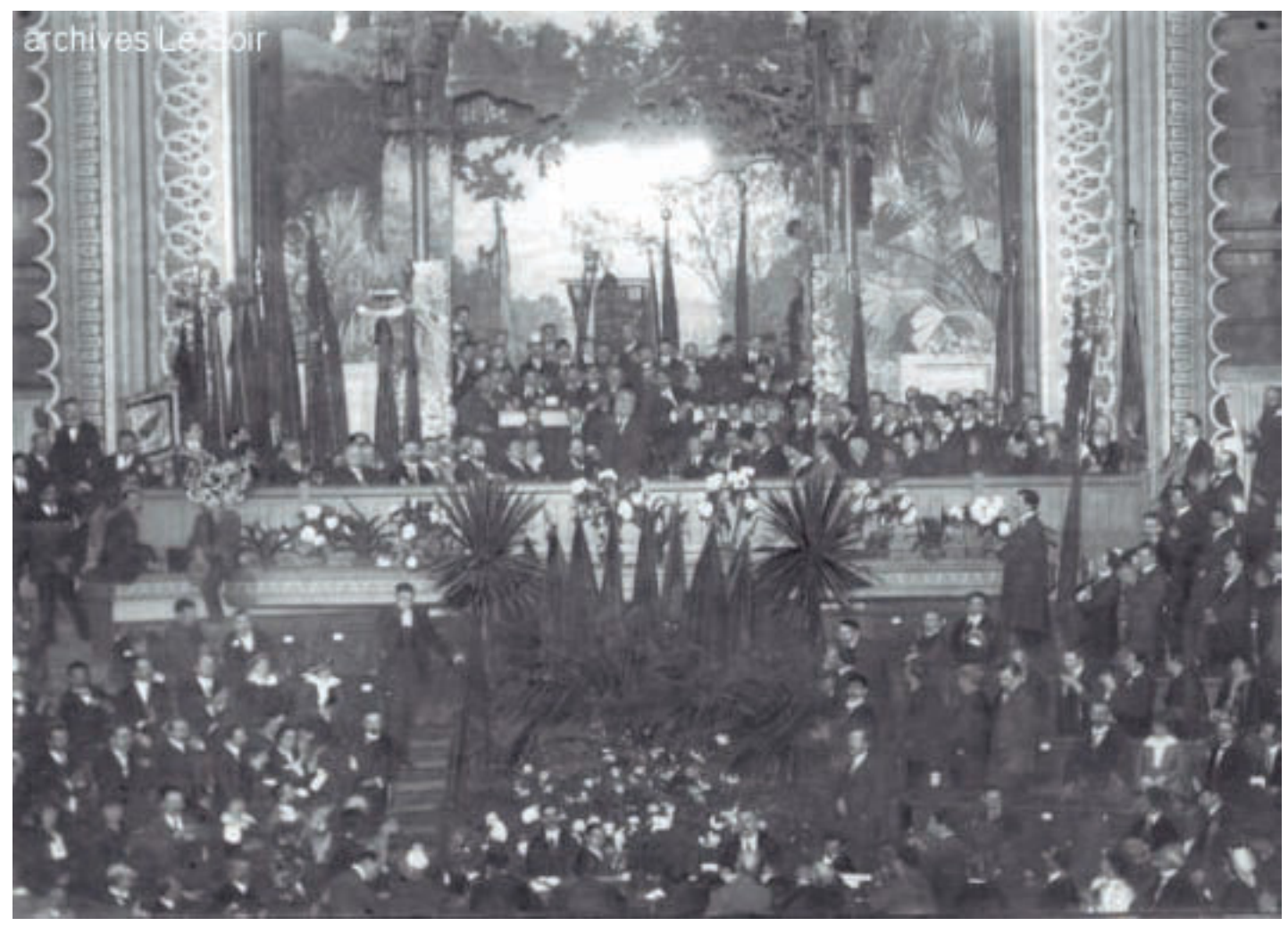

Entre le discours de 25 juillet et celui de 29 , Jean Jaurès modifie toutefois sa position par rapport au gouvernement français. Le 25, il le rend responsable de la dérive vers la guerre, sur le même plan que les autres puissances impérialistes. Tandis que le 29, le même gouvernement est décrit comme le meilleur allié de la paix.

S'agit-il d'une adaptation tactique ou d'une approche fondamentale? Le lendemain, Jaurès prend le train Bruxelles-Paris. Le 31 juillet, il rédige l'éditorial de L'Humanité "ang-froid nécessaire", demandant "aux hommes des nerfs d'acier [...] c'est l'intelligence du peuple de refouler les paniques, dominer les énervements et surveiller la marche des hommes et des choses, pour écarter de la race humaine l'horreur de la guerre"9 Il va ensuite manger au Café du croissant où l'attend le "patriote" Raul Vilain... qui le tue de deux balles dans la tête.

Nous n'aurions jamais, hélas, une réponse précise à la question de savoir comment Jaurès aurait réagi à la guerre et à $l^{\prime}$ "Union sacrée" à laquelle souscriront les socialistes.

En Allemagne, le $1^{\text {er }}$ août 1914, jour de la déclaration de guerre à la Russie, Hernan Muller assure que le SPD ne votera pas au Reichstag les crédits de guerre que demande le gouvernement. Plus, il part à Paris pour convenir d'une politique avec les socialistes français. 


\section{Atmosphère d'excitation guerrière}

Si les efforts de certains socialistes en vue d'arrêter l'engrenage sont sans doute notables, il faut mesurer le poids relatif de ceux-ci face à un raz-de-marée patriotique et belliciste.

En Allemagne, se développent des mouvements "pangermaniques" qui avancent des arguments en apparence "scientifiques", prétendant que le peuple allemand se multiplie si rapidement qu'il a donc un besoin pressant de nouveaux territoires. Et -affirment-ils-, un peuple ne peut se maintenir que s'il accroît sa population et son territoire: "quelqu'un doit faire place: ou les Slaves de l'Ouest et du Sud, ou bien nous". En conclusion, les peuples sont condamnés à se combattre. Le peuple allemand doit utiliser son armée, qui a prouvé son efficacité contre la France en 1870, pour obtenir de nouveaux territoires. ${ }^{10}$

En France, le sentiment "revanchard", qui revendique la guerre pour récupérer l'Alsace-Lorraine perdue en 1870, s'empare d'une partie croissante de l'opinion.

Au moment de l'annonce des déclarations de guerre, des documents photographiques témoignent du climat émotionnel et de la fièvre patriotique qui s'instaure dans les grandes villes des pays qui s'apprêtent à s'affronter. Meetings patriotiques, chasse aux prétendus espions, hymnes militaires chantés par d'immenses foules... Lorsque Trotsky quitte Vienne pour la Suisse pour éviter l'ordre d'internement aux Russes et aux Serbes, observe que la guerre avait donnée aux pays des apparences de fête. ${ }^{11}$ Dans une atmosphère d'excitation guerrière et de gigantesques manifestations chauvines, des foules de jeunes, souvent volontaires, proclament leur disposition à se ruer au front et à sacrifier leurs vies pour la patrie.

Dans ce contexte, les discours rationnels qui tentent d'avertir de l'ampleur de la tragédie proche, et qui revendiquent la solidarité de classe entre travailleurs de tous les pays, sont perçus comme la plus haute des trahisons.

\section{Les partis sociaux-démocrates adhèrent à "l'union sacrée"}

Le 2 août tout bascule. L'exécutif du SPD est divisé sur la question du vote des crédits militaires, réclamés par le chancelier Bethmann-Hollweg, au Reichstag. Le lendemain, à la réunion du groupe parlementaire, les "pro-guerre", animés par Scheidemann, le secrétaire du parti, attaquent : il faut voter les crédits pour préserver les libertés menacées par l'autocratie russe et l'impérialisme anglais. Et, chose jamais vue au SPD, ils sont résolus à rompre la discipline de vote sur un dossier d'une telle importance. Les "anti-guerre", eux, postulent, au nom des principes historiques du parti, un vote hostile aux crédits de guerre. Finalement, par 78 votes contre 14 les députés SPD décident de voter les crédits. La minorité se rallie par discipline au vote majoritaire.

Même si la base socialiste n'a pas été consultée sur cette question fondamentale, la décision du SPD de voter la guerre, ne rencontre aucune résistance ouvrière, sous forme de manifestation, grève, pétition ou refus d'accepter les ordres de mobilisation.

Rosa Luxemburg convoque chez-elle, à Berlin, plusieurs dizaines de militants 
pour rédiger un texte d'opposition à la guerre. N'y arrivent que sept. Fin août, Karl Liebknecht, leader des députés socialistes opposés à la guerre, se rend en Belgique, pays clairement agressé par l'Allemagne. À son retour, il dénonce les atrocités perpétrées par l'armée d'occupation. Il est privé de parole et menacé d'exclusion du parti.

Le retournement soudain du SPD, le parti "modèle" de l'IOS, considéré comme un exemple d'internationalisme prolétarien, aura un effet certain sur les autres partis sociaux-démocrates. Les socialistes anglais et français prendront le même chemin.

En France la situation est similaire. Léon Jouhaux, secrétaire général de la Confédération générale du travail (CGT), le grand syndicat socialiste, annonce dans le discours prononcé devant le cercueil de Jean Jaurès, le 4 août 1914, le ralliement des socialistes et de la CGT à l'union sacrée :

Jaurès a été notre réconfort dans notre action passionnée pour la paix. Ce n'est pas sa faute, ni la nôtre, si la paix n'a pas triomphé. Avant d'aller vers le grand massacre, au nom des travailleurs qui sont partis, au nom de ceux qui vont partir, dont je suis, je crie devant ce cercueil toute notre haine de l'impérialisme et du militarisme sauvage qui déchaînent l'horrible crime.

Cette guerre, nous ne l'avons pas voulue, ceux qui l'ont déchaînée, despotes aux visées sanguinaires, aux rêves d'hégémonie criminelle, devront en payer le châtiment. ${ }^{12}$

Les travailleurs, selon Jouhaux, partiront donc en guerre contre des " despotes " étrangers, seuls responsables du conflit.

Seuls quelques partis socialistes, comme ceux d'Italie, de Bulgarie, des Etats-Unis, de Pologne (alors inexistante comme nation) de Russie et de la Serbie ont résisté à la vague de patriotisme qui a emporté l'opinion. Ces derniers, dans un pays envahi, dénoncent l'invasion, mais refusent de soutenir le gouvernement. ${ }^{13}$

Comment expliquer la passivité initiale des travailleurs allemands, français et d'autres nations devant la guerre? Pierre Broué fournit une réponse intéressante :

Face à la menace d'arrestation et de jugement sommaire, l'ouvrier socialiste qui exprimerait son opposition à la guerre non seulement est privé de l'appui de son organisation, mais encore la trouve du même côté que son adversaire de classe. En fait l'attitude des 'masses' ne peut être posée indépendamment de celle des "chefs". ${ }^{14}$

Depuis trois générations, les travailleurs allemands avaient pour tradition d'agir dans le cadre des actions conçues et organisées au sein du SPD. L'action contre la guerre ne pouvait être qu'internationale, dans tous les pays concernés, tâche que seule l'IOS pouvait envisager. Convoqués par leurs organisations historiques, ils auraient peut-être exprimé leur opposition à la guerre. Mais, dépourvus de leur organisation, pourquoi devaient-ils agir seuls, pratiquement sans chances de succès, mais sûrs que une telle action serait fortement réprimée?

Ce n'est qu'en septembre que deux journaux suisses publient l'information: en Allemagne, quelques sociaux-démocrates s'opposent à la guerre. C'est en effet en décembre 1914 qu'un groupe restreint de dirigeants socialistes fera circuler le premier document critiquant la politique d" "union sacrée". En 1915, ils publient Die Internationale, un petit périodique qui sera interdit et circulera clandestinement. ${ }^{15}$ 


\section{Le refus de Karl Liebknecht de voter des nouveau crédits de guerre}

Le 2 décembre, devant le vote de nouveaux crédits de guerre au Reichstag, Karl Liebknecht rédige un projet de déclaration qui est rejeté par le groupe socialiste. Mais cette fois il vote contre les crédits.

Son argumentation deviendra la base idéologique du mouvement opposé à la guerre. Ces idées seront développées par Rosa Luxemburg, en 1915, quand, en prison, elle rédige la brochure La Crise de la social-démocratie, connue comme la brochure Junius. Elle sera publiée clandestinement en 1916.

Cette argumentation postule qu'il s'agit d'une guerre impérialiste puisqu'elle a pour but des conquêtes ; la neutralité du Luxembourg et de la Belgique a été violée et la population civile des territoires occupés souffre de traitements cruels, des localités entières ayant été dévastées.

La guerre n'a pas été déclenchée pour le bien du peuple allemand; ce n'est pas une guerre pour la défense du territoire et de la liberté. Les peuples n'ont pas été consultés : les gouvernements allemand et autrichien agissent dans l'ombre du semi-absolutisme et de la diplomatie secrète.

La guerre ne défend pas non plus la "civilisation" contre le tsarisme puisque le gouvernement allemand n'a pas hésité à aider le Tsar contre la révolution en Russie. La libération du peuple russe doit être l'œuvre du peuple russe lui-même.

Cependant, les libertés de presse et de réunion sont supprimées par l'instauration de l'état de siège.
Liebknecht demande une réglementation internationale humanitaire pour améliorer le traitement des prisonniers et le rejet du principe des représailles; l'opposition à toute annexion qui heurte le droit des peuples à disposer d'eux-mêmes. Seule une paix née sur la base de la solidarité internationale peut être une paix sûre. ${ }^{16}$

\section{Conclusions}

Jusqu'en juillet 1914, pratiquement tous les partis membres de $l^{\prime} I O S$ restent fidèles à leurs positions historiques d'opposition à la guerre. Du moins en apparence. La presse socialistes allemande, française, italienne, autrichienne et $\mathrm{d}$ 'autres pays en témoigne: la guerre est condamnée haut et fort et rien ne permet de percevoir l'adhésion au "patriotisme" qui suivra.

Cependant, le soudain revirement de l'antimilitarisme à l'union-sacrée ne concerne pas tous les partis sociaux-démocrates. L'allemand, le belge, le britannique et français, basculent rapidement, tandis que les socialistes bulgares, italiens, russes (bolcheviks et mencheviks) et serbes, continuent à manifester leur opposition à la guerre, même dans des circonstances difficiles.

Comment expliquer telle différence?

La décision de soutenir leur gouvernement en guerre est prise par les dirigeants politiques et syndicaux des grands partis allemand, français, anglais, et belge, qui avaient bien plus que leurs "chaînes à perdre". D'une part, la guerre semple inéluctable ; même des manifestations massives à l'échelle européenne n'avaient que des faibles chances de l'éviter. Et, d'autre part, ces 
puissants partis risquent illégalité et clandestinité ce qui implique la perdre de leur place dans l'État, c'est-à-dire des parlementaires, périodiques, maisons culturelles, syndicats, et bien d'autres organisations.

Les partis socialistes qui restent attachés à leurs principes sont ceux qui disposent d'un appareil réduit (italiens, serbes), ceux qui sont dans la clandestinité (bulgares, russes), ou ceux dont le pays ne participe pas au conflit.

Finalement, les socialistes opposés à la guerre, venant de pratiquement tous les pays belligérants ou non belligérants (allemands, français, russes, italiens, britanniques, suisses, suédois, norvégiens, néerlandais, polonais, roumains, bulgares ainsi et du Bund ${ }^{17}$ ), vont se rencontrer du 5 au 8 septembre 1915 en à Zimmerwald (Suisse), pour organiser une courante socialiste opposée à la guerre, qui présage les futurs partis communistes.

\section{Resumo}

Durante o seu quarto de século de existência, a Internacional Operária Socialista (IOS ou Segunda Internacional), manifestou constantemente oposição ao militarismo e da guerra, exceto nos últimos dias antes do conflito. De certa forma, talvez surpreendentemente, os principais partidos socialistas abandonaram suas posições históricas e reuniram-se para a "união sagrada", cada um apoiando o esforço de guerra em seu país. Salvo algumas exceções. Neste artigo, primeiro gostaria de traçar alguns aspectos da posição histórica dos socialistas sobre a guerra; Então vamos fazer a história de sua recuperação, avançando para terminar alguma explicação.

Palavras-chave: Social-democracia ; união sagrada ; antimilitarismo ; Segundo Internacional.

\section{Abstract}

During his quarter-century of existence, the International Socialist Workers (IOS or Second International), has consistently expressed opposed views to militarism and war, except in the last few days before the conflict. In a way perhaps surprisingly, the major socialist parties abandoned their historical positions and rallied to the "sacred union", each supporting the war effort in their country. There were some exceptions. In this article we first wish to trace some aspects of the historical position of the Socialists about the war; Then we'll do the story of their turnaround, advancing to finish some explanation.

Keywords: Social-democracy; sacred union; anmilitarism; Second International.

\section{Notas}

1 Broué, 1971, 24-27.

2 Rosa Luxembourgo, 1976, 73.

3 Broué, 1971, 20-21.

4 Internationale et patrie sont liées, in Une vie, une œuvre, 2014, 51.

5 Publié à www.assemblee-nationale.fr/histoire/ jaures/discours/responsables-guerre_25071914. asp.

6 Principal journal du Parti social-démocrate des travailleurs d' Allemagne, fondé à Leipzig en 1876.

7 Sur la réunion de l'IOS à Bruxelles et l'intervention de Jaurès : http://dormirajamais.org/jaures-1/. 
8 Archives du journal Le Soir.

9 Sang-froid nécessaire, in Une vie, une cuvre, 2014, 53

${ }^{10}$ Simard \& Laville, 2000, 334.

${ }_{11}$ Trotsky, 1953, 275.

${ }^{12}$ http://www.histoiresocialedeslandes.org/p3 impact_win02.asp

${ }_{13}$ Novack, 1977, 96.

${ }^{14}$ Broué, 1971, 60.

${ }^{15}$ Rosa Luxembourg, 1976, 56.

${ }^{16}$ Publié dans: http://www.jpmarat.de/francais/ kldecf.html.

17 Socialistes juifs en Europe de l'Est.

\section{Sources}

Broué Pierre, 1972, Révolution en Allemagne, 1917-1923, Éditions de minuit

Luxemburgo Rosa, 1976, Obras escogidas, Ed Pluma, Bogotá

Novack George, Frankel Dave, Feldman Fred, 1977, Las tres primeras internacionales, su historia y sus lecciones, Ed Pluma, Bogotá

Simard Marc \& Laville Christian, 2000, Histoire de la civilisation occidentale, Ed Renouveau pédagogique, Québec.

Une vie, une cuvre. Jean Jaurès, avril 2014, cahier hors-série du journal Le Monde

Trotski Léon, 1953, Ma vie, Ed Gallimard 